\title{
Cryogenic Preamplifiers for Magnetic Resonance Imaging
}

Johansen, Daniel H.; Sanchez-Heredia, Juan D.; Petersen, Jan R.; Johansen, Tom K.; Zhurbenko, Vitaliy; Ardenkjaer-Larsen, Jan $\mathrm{H}$.

Published in:

IEEE Transactions on Biomedical Circuits and Systems

Link to article, DOI:

10.1109/TBCAS.2017.2776256

Publication date:

2018

Document Version

Peer reviewed version

Link back to DTU Orbit

Citation (APA):

Johansen, D. H., Sanchez-Heredia, J. D., Petersen, J. R., Johansen, T. K., Zhurbenko, V., \& Ardenkjaer-Larsen, J. H. (2018). Cryogenic Preamplifiers for Magnetic Resonance Imaging. IEEE Transactions on Biomedical Circuits and Systems, 12(1), 202-210. [8233404]. https://doi.org/10.1109/TBCAS.2017.2776256

\section{General rights}

Copyright and moral rights for the publications made accessible in the public portal are retained by the authors and/or other copyright owners and it is a condition of accessing publications that users recognise and abide by the legal requirements associated with these rights.

- Users may download and print one copy of any publication from the public portal for the purpose of private study or research.

- You may not further distribute the material or use it for any profit-making activity or commercial gain

- You may freely distribute the URL identifying the publication in the public portal

If you believe that this document breaches copyright please contact us providing details, and we will remove access to the work immediately and investigate your claim 


\title{
Cryogenic Preamplifiers for Magnetic Resonance Imaging
}

\author{
Daniel H. Johansen, Juan D. Sanchez-Heredia, Jan R. Petersen, Tom K. Johansen, Vitaliy Zhurbenko, and \\ Jan H. Ardenkjær-Larsen.
}

\begin{abstract}
Pursuing the ultimate limit of detection in magnetic resonance imaging (MRI) requires cryogenics to decrease the thermal noise of the electronic circuits. As cryogenic coils for MRI are slowly emerging cryogenic preamplifiers are required to fully exploit their potential. A cryogenic preamplifier operated at $77 \mathrm{~K}$ is designed and implemented for ${ }^{13} \mathrm{C}$ imaging at $3 \mathrm{~T}$ (32.13 MHz), using off-the-shelves components. The design is based on a high electron mobility transistor (ATF54143) in a common source configuration. Required auxiliary circuitry for optimal cryogenic preamplifier performance is also presented consisting of a voltage regulator (noise free supply voltage and optimal power consumption), switch and trigger (for active detuning during transmission to protect the preamplifier). A gain of $18 \mathrm{~dB}$ with a noise temperature of $13.7 \mathrm{~K}$ is achieved. Performing imaging experiments in a 3T scanner showed an $8 \%$ increased signal-to-noise ratio (SNR) from 365 to 399 when lowering the temperature of the preamplifier from $296 \mathrm{~K}$ to $77 \mathrm{~K}$ while keeping the coil at room temperature. This work thus enables the merger of cryogenic coils and preamplifiers in the hopes of reaching the ultimate limit of detection for MRI.
\end{abstract}

Index Terms-Biomedical electronics, cryogenic electronics, hyperpolarization, Low noise amplifier, magnetic resonance imaging, preamplifiers.

\section{INTRODUCTION}

Combining anatomical proton $\left({ }^{1} \mathrm{H}\right)$ magnetic resonance imaging (MRI) and hyperpolarized metabolic carbon $\left({ }^{13} \mathrm{C}\right)$ magnetic resonance spectroscopic imaging (MRSI) yields a superlative clinical diagnostic tool for investigation cancer aggressiveness and treatment response much earlier than with other non-invasive imaging procedures [1], [2]. One of the primary concerns of MRI is the inherently limited signal-to-noise ratio (SNR) impairing image quality (making it harder for clinicians to evaluate results) and acquisition speed (increasing scanner time and thus cost) [3]. Further, for hyperpolarization due to the transient nature of the experiment, higher baseline SNR is invaluable because averaging is generally not viable since it would involve multiple injections of contrast agent [4]. To approach the ultimate limit of detection in MRI and especially for applications of hyperpolarization, cryogenics are

Manuscript received July 4th, 2017; revised October 23rd, 2017. This work was supported in part by the Danish National Research Foundation under grant DNRF124.

The authors are with the Department of Electrical Engineering, Technical University of Denmark, Kgs. Lyngby, Denmark. D. H. Johansen and V. Zhurbenko are with the Center for Hyperpolarization in Magnetic Resonance and Electromagnetic Systems. J. D. Sanchez-Heredia and J. R. Petersen are with the Center for Hyperpolarization in Magnetic Resonance. T. K. Johansen is with Electromagnetic Systems. *J. H. Ardenkjær-Larsen is with the Center for Hyperpolarization in Magnetic Resonance and GE Healthcare, Brøndby, Denmark (correspondence e-mail: jhar@elektro.dtu.dk). paramount. Cryogenically cooled receive coils are receiving a lot of attention in the form of either copper or high temperature superconducting (HTS) surface coils. However, design and implementation of cryogenic preamplifiers and required auxiliary systems have largely been omitted within the MRI community.

A clinical MRI system consist of two primary magnetic fields. The main field $B_{0}$ is a static magnetic field generally ranging from 1.5 to $7 \mathrm{~T}$, which create a proportional weak polarization of the nuclear spins of the patient. The primary nuclear spin in a clinical setting is ${ }^{1} \mathrm{H}$. In the hyperpolarized regime many different nuclei can be imaged such as ${ }^{129} \mathrm{Xe}$, ${ }^{3} \mathrm{He},{ }^{15} \mathrm{Ni}$ or ${ }^{13} \mathrm{C}$ [5]. Here focus is limited to ${ }^{13} \mathrm{C}$. The precession (resonance) frequency of the nuclear spins is the product of the main field strength and the gyromagnetic ratio of the nuclei. By applying an RF pulse (a second magnetic field) orthogonally to the main field at the Larmor frequency of the nuclei of interest tilts them away from equilibrium. When the RF pulse is switched off the nuclei relax back to equilibrium. The precession is then captured by Faraday induction [6], [7].

Using cryogenics to improve SNR within nuclear magnetic resonance spectroscopy (NMR) and MRI is well known. H. Kovacs et al. [8] thoroughly reviews the history of cryogenic probes for NMR. L. Darrasse et al. [9] reviewed cryogenic biomedical MRI probes. Within biomedical MRI the largest breakthrough so far is achieved by Bruker with their CryoProbe for preclinical small animal studies [10], [11].

Publications and commercial products for room temperature preamplifiers for MRI is plentiful. C. Lim et al. [12] describes three designs for $619 \mathrm{MHz}$. S. Yadav [13] presents simulations of a cascaded topology for $64 \mathrm{MHz}$. F. Doty et al. [14] provides a topology for $300 \mathrm{MHz}$ excluding stabilization components. Several vendors source room temperature MRI preamplifiers e.g. WanTcom [15], Teledyne e2v [16] and HiQ.A. Inc. [17].

The focus of this work is cryogenic preamplifiers for MRI where publications are very sparse. M. Pospieszalski has written a general review on cryogenic amplification using field effect transistors [18]. Cryogenic preamplifiers are used in a range of different applications ranging from the deep space network [19] over SQUIDs [20], [21] and proof-ofconcepts [22]-[24] to NMR [25]-[28]. For MRI applications, J.G. van Heteren et al. [29] mentions the use of a cryogenic preamplifier based on a JFET for $2.7 \mathrm{MHz}$ cooled to $77 \mathrm{~K}$. However, no design is supplied and thus reproducing is not possible. F. Resmer et al. [30] provides a design for $425 \mathrm{kHz}$ 
MRI also based on a JFET operating at $77 \mathrm{~K}$. Hence the field of cryogenic preamplifiers for MRI is a sparse field with only a few publications aimed at low main magnetic field applications and excluding a complete design.

The primary contributions of this work is the design and implementation of a cryogenic preamplifier for MRI based on an enhancement mode pseudomorphic high electron mobility transistor (pHEMT) utilizing a common source topology cooled to $77 \mathrm{~K}$ using liquid nitrogen for ${ }^{13} \mathrm{C}$ imaging at $3 \mathrm{~T}$ (32 MHz). Furthermore, no current literature describe required auxiliary systems when using preamplifiers in a clinical MRI scanner. The auxiliary systems contains circuitry such as voltage regulators and active detuning switching for protecting the preamplifier. Hence, an additional contribution of this work is the description of auxiliary circuitry used for both room temperature and cryogenic preamplifiers.

\section{SYSTEM OVERVIEW}

Before exploring the intricacies of developing cryogenic preamplifiers the system architecture is presented. Apart from the coils generating the main, gradient and RF fields the general system is seen in Fig. 1. It is a receive only system based on a volume coil with attached matching and active detuning network. Following the coil and matching circuit is the preamplifier placed inside a cryostat. The cryostat is cooled to $77 \mathrm{~K}$ using liquid nitrogen. Separating the cryogenic preamplifier from the coil is an unusual constellation. Normally the coil and matching circuit would also be cooled. However, only the preamplifier is cooled because it is the primary focus of this work. This also makes characterization of the cryogenic preamplifier much easier since the input and output terminals are at room temperature. The matching and active detuning circuit is described by J. D. Sanzhez-Heredia et al. [31].

The auxiliary circuitry consists of the regulator, trigger and switch. In order to optimize the power consumption for a given preamplifier a voltage regulator is usually needed. Also, the voltage regulator further filters the supply signal. An active detuning signal is also supplied from the scanner. A high

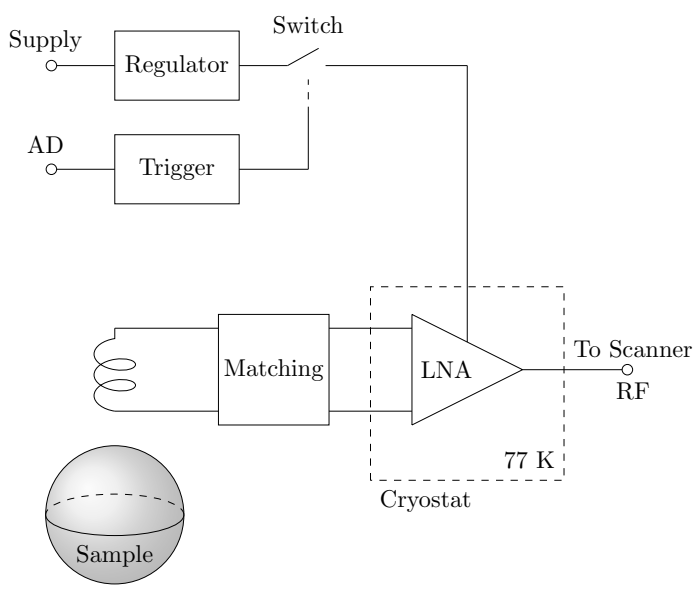

Figure 1. Overview of the system. The regulator, switch and trigger constitute the auxiliary system. The low noise preamplifier (LNA) is operated at $77 \mathrm{~K}$ while the coil is kept at room temperature.

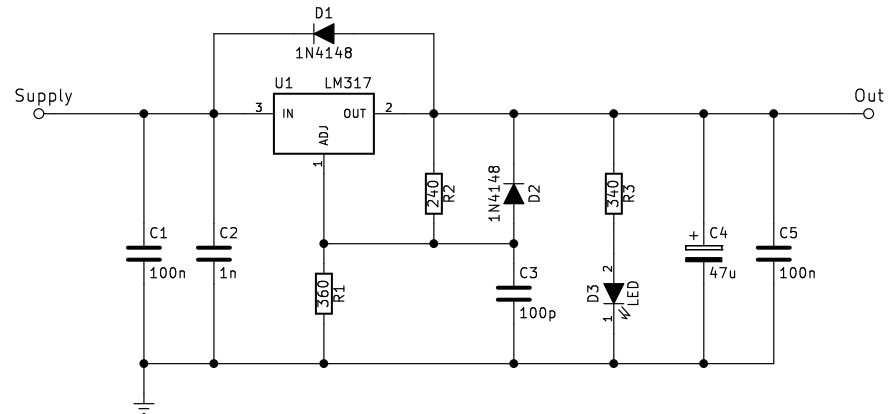

Figure 2. Schematic of the voltage regulator [32]. This is a classic design where $\mathrm{D} 3$ and $\mathrm{R} 3$ is added to act as a state indicator and to draw a small current which lock the output voltage of the LM317 which otherwise is floating.

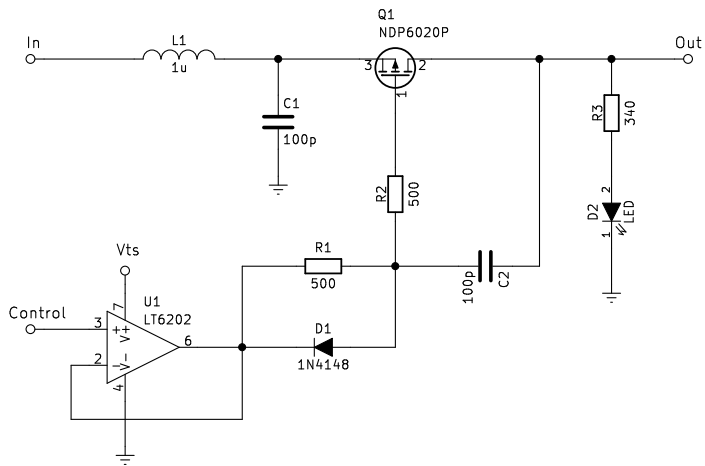

Figure 3. Schematic of the fast supply switch based on a P-channel MOSFET acting as the supply switch. It is controlled by the signal from the trigger which is buffered by U1 in order to ensure proper current sourcing and sinking during switching.

voltage level indicates that RF transmission is commencing. A low voltage indicates that the scanner is ready to receive. Hence, at a high active detuning voltage the switch needs to disengage the supply voltage to the preamplifier in order to minimize the impact of the high powered RF pulse. The trigger thus acts both as an inverter and a filter.

\section{A. Voltage Regulator}

The voltage regulator circuit is based on an LM317 linear regulator and seen in Fig. 2 [32]. Here the capacitors C1, C2, $\mathrm{C} 4$ and $\mathrm{C} 5$ ensures stability and filters unwanted noise. The diodes D1 and D2 are overvoltage protection. The resistors $\mathrm{R} 1$ and R2 determine the output voltage. If no current is drawn from the regulator the output voltage is undefined and could cause problems when the preamplifier is off during transmission. The resistor R3 and LED D3 are loading the regulator with a small current to ensure that the output voltage remains constant even with no load.

\section{B. Switch}

The switch, Fig. 3, is designed around a P-channel MOSFET Q1. To control and limit the preamplifier inrush current the transistor turn on must be controllable and reproducible. This is done by increasing the transistors Miller capacitance via $\mathrm{C} 2$. With $\mathrm{R} 2$ working as a current source, driving $\mathrm{C} 2$ and 


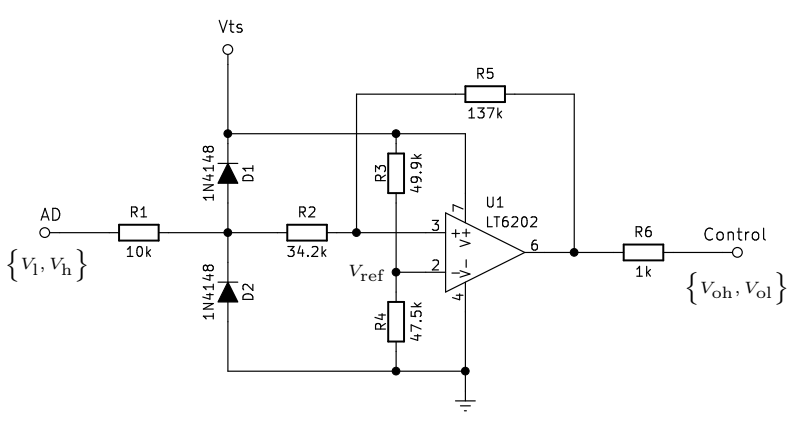

Figure 4. Schematic of the trigger circuit. It is a non-inverting Schmitt trigger topology with input protection in the form of R1, D1, and D2.

the transistor gate. The transistor thus ramps the output voltage up linearly. Turn off is fast due to the diode D1, pulling the transistor gate to the positive supply rail, only limited by $\mathrm{R} 1$ and the operational amplifiers, U1, output current. R2 prevents the transistor from oscillating when turning on and off. L1 and C1 low-pass filters the power supply to the amplifier. R3 and D2 is a simple power on indicator.

\section{Schmitt Trigger}

The trigger circuit is seen in Fig. 4. It is a non-inverting Schmitt trigger topology using an LT6202 operational amplifier triggering a low output when the input voltage transitions above a threshold voltage $V_{\mathrm{h}}$. When the input transitions below $V_{1}$ the output is pulled high. The diodes D1 and D2 prevent the input to the operational amplifier from going above or below the supply rails by more than the knee voltage of the diodes. The series resistor R1 limits the current and is chosen such that only a few hundred micro amperes are drawn from the active decoupling circuit at maximum applied voltage. This also protects the diodes from failure by limiting the current they can source or sink. The non-inverting Schmitt trigger is described by

$$
\begin{aligned}
R 1+R 2 & =\frac{R_{5}\left(V_{1}-V_{\mathrm{h}}\right)}{V_{\mathrm{oh}}-V_{\mathrm{ol}}}, \\
V_{\mathrm{ref}} & =\frac{V_{\mathrm{h}} V_{\mathrm{ol}}-V_{1} V_{\mathrm{oh}}}{V_{\mathrm{h}}-V_{\mathrm{l}}-V_{\mathrm{oh}}+V_{\mathrm{ol}}},
\end{aligned}
$$

where $V_{\text {oh }}$ and $V_{\text {ol }}$ are the high and low output of the operational amplifier respectively. In this case the operational amplifier is a singly supply, rail to rail and thus the high voltage is equal to $\mathrm{Vts}$ and the low voltage is $0 \mathrm{~V}$. $V_{\text {ref }}$ is the voltage at pin 2 of $\mathrm{U} 1$ determined by the voltage division of $\mathrm{R} 3$ and R4. The voltage Vts is a regulated voltage fed from a separate regulator circuit identical to Fig. 2. This is to separate the switching supply from the more critical preamplifier supply voltage.

\section{PREAMPLIFIER DESIGN}

\section{A. Transistor}

Transistors are typically separated into two basic types; the bipolar junction transistor (BJT) and field effect transistor (FET). An NPN type BJT is made by sandwiching a P-type semiconductor between two N-type semiconductors. In the Ntype semiconductor the majority charge carriers are electrons, whereas in the P-type they are holes. Thus when a voltage (principally an electric field) is applied, electrons are injected from the emitter and into the base where they diffuse to the collector. In the base, being a P-type material, the electrons are minority charge carriers [33]. Minority charge carriers can only be thermally excited. Hence at low temperatures the electrons excited into the conduction band is essentially zero and thus freeze out occurs; the semiconductor becomes an insulator. At low temperatures the diffusion length, especially from base to emitter, becomes significantly impaired. Hence electron mobility in a BJT at low temperatures is very low and the current gain approaches unity [34], [35]. The story is somewhat the same for the PNP type BJT. Here the emitter and collector are P-type exhibiting freeze out, which leads to an even worse performance at low temperature [36]. During the late 1980s bandgap engineering lead to the invention of the heterojunction bipolar transistor (HBT). An HBT can be used at low temperatures but require specific design of the transistor [37]-[42]. Since a dedicated transistor process is usually not available, we draw the conclusion that bipolar transistors are generally not an option for low temperature electronic designs. This further excludes the use of many operational amplifiers since they, for the most part, employ a mixture of NPN and PNP type BJTs.

The conclusion is, luckily, the opposite for N-type FETs. Since electrons are majority charge carriers and excitation is dominated by the electric field in the gate, N-type FETs become less dependent on temperature. Indeed fewer electrons are excited at lower temperatures but this effect is easily compensated by increasing the gate voltage [36], [43]. A higher gate voltage entails a higher electrical field in the device. Hence, the voltage cannot be increased infinitely because the transistor first electrically collapses (avalanche effect) and finally physically breaks down. MOSFETs tend to not show good cryogenic performance due to trapping in the metal oxide resulting in a significantly higher flicker noise [36]. Further, P-type FETs do not function properly at low temperatures due to the electron transport being by minority charge carriers in the P-type material. Hence operating silicon based integrated circuits using FET technology at cryogenic temperatures is a challenge due to two reasons; usage of both $\mathrm{N}$ - and $\mathrm{P}$ type FETs and changed characteristics of the FET at lower temperature which cannot be compensated externally [44]. As BJTs evolved into HBTs so FETs developed into pHEMTs by use of bandgap engineering. A pHEMT is essentially an FET on steroids. In a pHEMT the electrons have a higher mobility yielding better gain and noise performance, especially at higher frequencies. Furthermore, having higher electron mobility decreases the freeze out temperature while still driven by field effect. Hence it should perform well at cryogenic temepratures.

\section{B. Topology}

The implemented topology consists of a pHEMT in a common source configuration with added stability measures as 


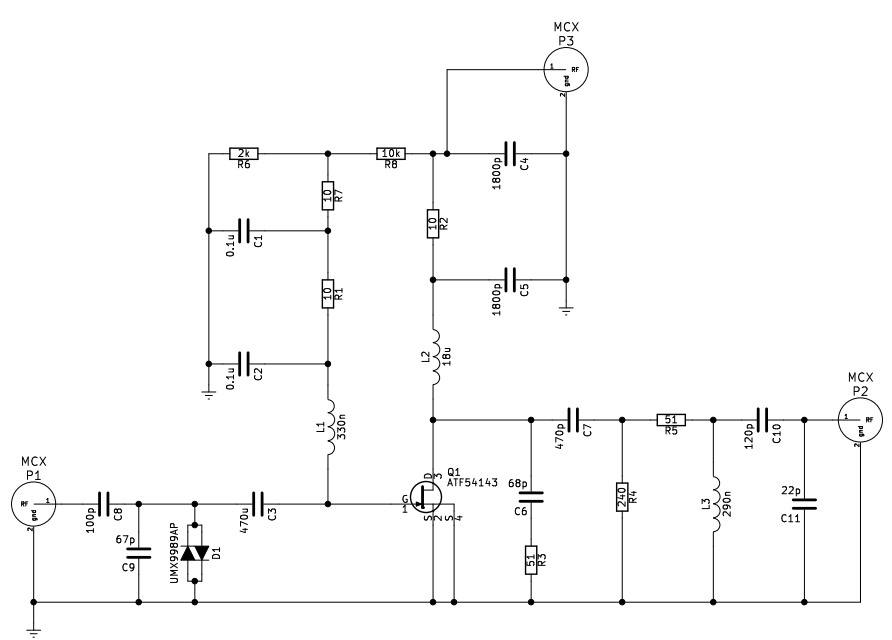

Figure 5. Schematic of the preamplifier. The design is based on a common source topology using feed inductors in both input and output. Stability is ensured primarily on the output by resistive attenuation.

seen in Fig. 5 [45]. The source of the transistor Q1 is connected to ground, the gate is used as a high impedance voltage input and the drain is a voltage controlled current output.

The stability of a two port network is determined by the Edward-Sinsky stability factor [46],

$$
\begin{aligned}
\mu & =\frac{1-\left|S_{11}\right|^{2}}{\left|S_{22}-S_{11}^{*} \Delta\right|+\left|S_{12} S_{21}\right|}>1, \\
\Delta & =S_{11} S_{22}-S_{12} S_{21},
\end{aligned}
$$

where $S_{x y}$ are the four S-parameters of the preamplifier as a function of frequency. Equation (3) represents the necessary and sufficient conditions for stability of a two port. The more frequently used Rollett stability factor $K$ constitutes only a necessary condition and must be paired with the condition $\Delta<1$ to ensure stability [47].

Seen from the gate of Q1 the components L1, C1, C2 and $\mathrm{R} 1$ constitute a high pass filter. The high pass filter on the input ensures low frequency stability according to (3). Furthermore, the inductor L1 is chosen such that the impedance at the gate (G1) is inductive. Hence, the series-shunt connected capacitors $\mathrm{C} 8$ and $\mathrm{C} 9$ match the optimal noise impedance of the preamplifier to $50 \Omega$. Resistors R6 and R8 determine the bias voltage. It is vital that the resistors are chosen such that the impedance seen by the high pass filter is high. If the biasing resistors alter the characteristics of the high pass filter oscillation might occur. The required bias voltage is found as a trade-off between the wanted noise and gain of the transistor. Especially at lower temperatures, as presented in the previous subsection, the bias voltage should be increased to maintain the same gain as at room temperature.

On the drain of Q1 is the feed inductor L2 which should be chosen such that the resulting impedance is high. Some designs employ a parallel capacitor across L2 resonant at the frequency of interest. L2 is preceded by a supply filter consisting of $\mathrm{C} 5, \mathrm{C} 4$ and $\mathrm{R} 2$ in order to make sure the voltage from the regulators are properly filtered. The size of $\mathrm{R} 2$ and the resistance of $\mathrm{L} 2$ implicitly determine the compression point

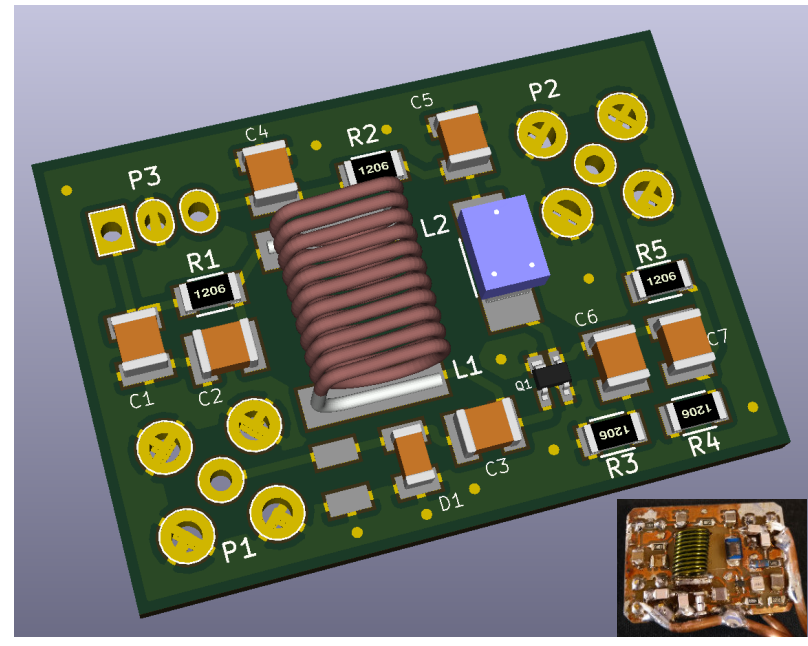

Figure 6. Example of preamplifier layout excluding biasing resistors, input and output matching. In the bottom right the preamplifier photo is shown where biasing, input and output matching has been reworked to fit the layout. The size of the PCB is $37 \mathrm{~mm} \times 26 \mathrm{~mm}$.

of the preamplifier by controlling the voltage at the drain of the transistor. Larger resistance results in lower voltage at the drain of Q1 hence the compression point is also lowered. The capacitors $\mathrm{C} 4$ and $\mathrm{C} 5$ should be chosen as large as possible but is a trade-off between blanking time of the preamplifier, since they increase the time constant of the filter and the resonance of the capacitors arising from internal parasitics.

The capacitor C6 and resistor R3 forms a low pass filter ensuring high frequency stability. Furthermore, resistors R4 and R5 form a broadband attenuator which also increase stability of the preamplifier. Capacitors $\mathrm{C} 3$ and $\mathrm{C} 7$ are simply DC blocks. The output matching is tuned by adjusting L3, C10 and C11. Finally, a set of UMX9989 crossed protection diodes D1 are placed at the input to short out any excess power from the transmitter.

The layout of the preamplifier is seen in Fig. 6. The primary aspect of the layout is grounding. Especially at the protection diodes and the transistor. If the transistor source is not properly grounded an inductor is effectively connected between the source and the ground. Hence a source degenerated topology arises. By simulation and experiment this was seen to make the preamplifier more low frequency stable, but more high frequency unstable.

\section{Passive Components}

Passive components for cryogenic operation are not readily available. Even though most passive components are rated in the -50 to 150 degree centigrade range they can be used at lower temperatures. Their value, however, will change by some significant amount. Given resistors of $200 \mathrm{ppm} / \mathrm{K}$ a corresponding $5 \%$ additional resistance occurs at $77 \mathrm{~K}$.

Capacitors, depending on the materials used for construction, can behave much different. Examples of X5R and Y5V type capacitors at $77 \mathrm{~K}$ shows not only an $80 \%$ decreased capacitance, but also a significant increase in equivalent series resistance (ESR). In brief, all capacitors operated at cryogenic 


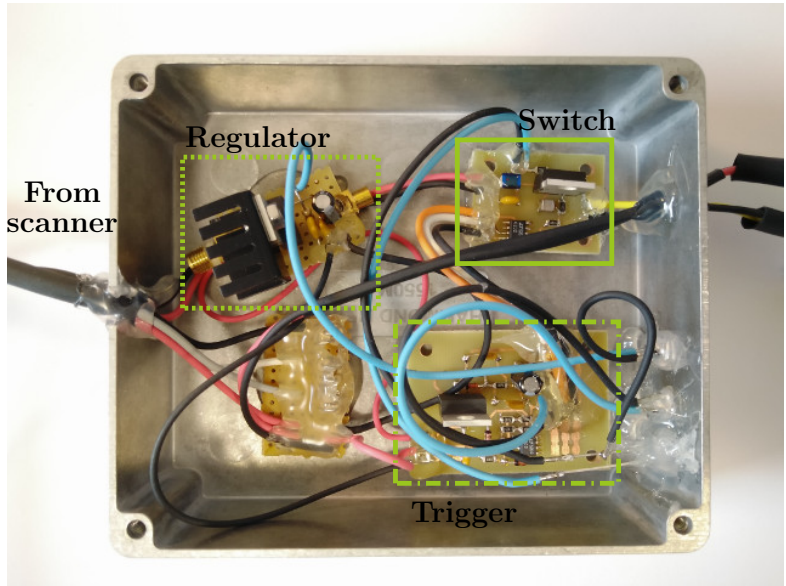

Figure 7. Auxiliary systems consisting of the regulator, trigger and switch enclosed in an aluminium box. The size of the aluminium box is $120 \mathrm{~mm} \mathrm{x}$ $100 \mathrm{~mm} \times 35 \mathrm{~mm}$.

temperatures should be NP0 type showing an approximate $3 \%$ capacitance increase whereas ESR is decreased [48], [49]. Hence the Q-factor is increased. This must be taken into account when designing the preamplifier.

\section{Measurement SetuP}

\section{A. Bench Characterization}

For bench characterization the preamplifier is disconnected from the coil in Fig. 1. The S-parameter matrix is measured using an Agilent ENA (E5062A) network analyzer by performing a two port measurement. In order to measure the noise an Agilent PSA (E4440A) spectrum analyzer with noise extension (346B) is used. For noise measurements the noise source is connected to the input of the preamplifier and the output is connected to the spectrum analyzer. The preamplifier and cryostat are placed in a shielded box (R\&S CMW-Z10) to attenuate spurious signals impairing the noise measurement.

\section{B. Verification in Scanner}

The system shown in Fig. 1 is tested in a scanner with a small animal (mouse) volume coil. In this work a clinical 3 $\mathrm{T}$ General Electric Signa HDx is used. To obtain images a chemical shift imaging (CSI) sequence is used with a fieldof-view of $120 \mathrm{~mm}$ x $120 \mathrm{~mm}$, slice thickness $10 \mathrm{~mm}$ and 16-by-16 points. The duration of each scan is $19 \mathrm{~s}$ and 128 averages is used. Thus resulting in a scan time of just under $41 \mathrm{~min}$. A $1 \mathrm{M}^{13} \mathrm{C}$-bicarbonate spherical phantom, $37.5 \mathrm{~mm}$ in diameter, is used. The phantom corresponds electrically to a rat.

\section{RESULTS}

\section{A. Auxiliary Systems}

The supply voltage from the scanner is $10 \mathrm{~V}$. The voltage regulator design in Fig. 2 outputs $3.15 \mathrm{~V}$. The nominal supply voltage for the preamplifier is $3 \mathrm{~V}$. The extra $0.15 \mathrm{~V}$ is dropped in the LC filter prior to Q1 in Fig. 3. The trigger is designed using a supply voltage of $3.15 \mathrm{~V}$ supplied from another regulator than the one for the preamplifier (not explicitly shown). The active detuning signal levels are $7 \mathrm{~V}$ and $-5 \mathrm{~V}$. The high threshold voltage is chosen as $1 \mathrm{~V}$ and the low threshold voltage to $2 \mathrm{~V}$. By choosing R5 (or R2) in Fig. 4 the reference voltage and R2 (or R5) is determined. Knowing the reference voltage, it is a simple task to determine R3 and R4. Lastly, the switch Fig. 3 is designed to have an approximate $4 \mu$ s turnon time verified by Spice simulation. The auxiliary systems are seen in Fig. 7. The auxiliary systems presented are not designed using non-magnetic components. Since the auxiliary systems are placed in an aluminium box, in the fringe field of the main magnet, far from region of interest, this has not posed an issue.

\section{B. Preamplifier}

The ${ }^{13} \mathrm{C}$ Larmor frequency at $3 \mathrm{~T}$ is $32.115 \mathrm{MHz}$. Hence the preamplifier is designed for this frequency. The printed circuit board (PCB) is fabricated using an in-house facility. A standard double sided FR4 is used with epsilon 4.3, copper thickness $35 \mathrm{um}$ and thickness $1.6 \mathrm{~mm}$. The design is seen in the bottom right of Fig. 6. The fabricated PCB size is $37 \mathrm{~mm}$ x $26 \mathrm{~mm}$.

The transistor used here is the Broadcom (formerly Avago) ATF54143. The choice of the transistor is in part due to showing the best noise performance of commercially available transistors. In part because it is an enhancement mode pHEMT. An enhancement mode pHEMT does not require a negative gate voltage to turn off. This is preferred because a negative gate voltage does not have to be generated from the single supply of the scanner. The capacitors used are PPI high-Q having an inherent tolerance of $5 \%$.

The bias voltage was chosen as $0.5 \mathrm{~V}$ which yields a fair trade-off between noise and gain of the transistor at both room and cryogenic temperature. The bias voltage is kept fixed for the room temperature and cryogenic measurements. The measured bias voltage is $0.54 \mathrm{~V}$ at room temperature.

The S-parameters are shown in Fig. 8. The input reflection coefficient in Fig. 8a shows that the preamplifier is highly mismatched. Even though the input impedance is highly mismatched the amplifier is matched primarily to decrease noise. The simulated and measured room temperature $S_{11}$ does differ significantly when in this high mismatch regime. Further, at $77 \mathrm{~K}$ the mismatch is increased even further. The input reflection coefficient is seen to increase when cooled in Fig. 8a. This is partly due to the decreased current draw. Partly that the transistor input impedance increases at lower temperatures. The reverse isolation in Fig. 8b shows fair correlation between simulation and measurement. At $77 \mathrm{~K}$ the isolation is unchanged. Fig. 8c shows the gain where the simulated and measured data at $296 \mathrm{~K}$ are well correlated. When cooled the gain decreases by approximate $5 \mathrm{~dB}$. Having the bias voltage fixed the decrease in gain is expected at lower temperatures and can be mitigated by increasing the bias voltage. The output reflection coefficient also shows a fair correlation between simulation and measurement and a minor offset at $77 \mathrm{~K}$.

The stability of the preamplifier is assessed using three different approaches. The first is to evaluate (3) from the 


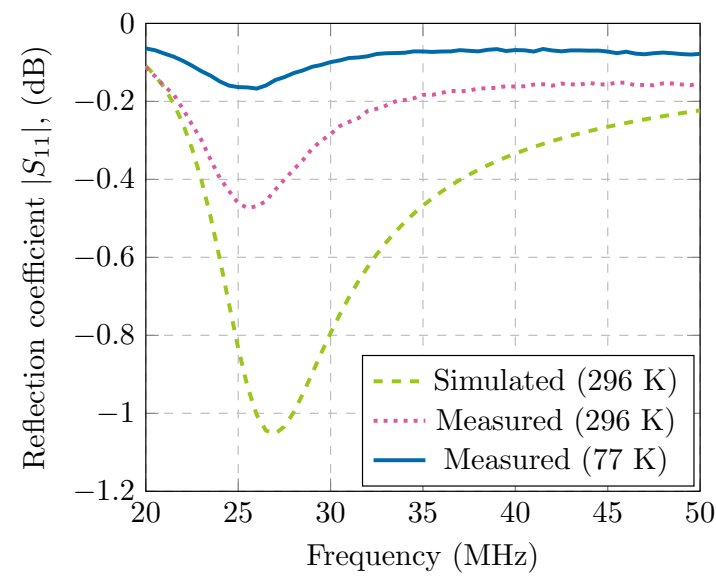

(a)

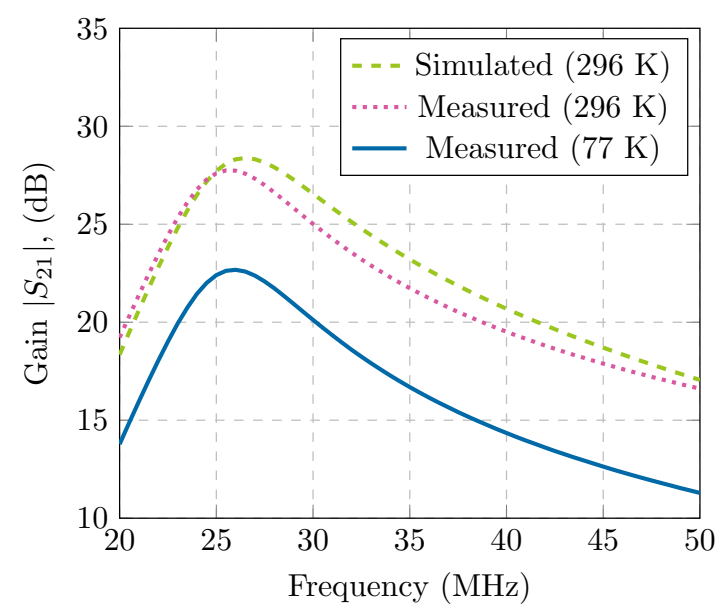

(c)

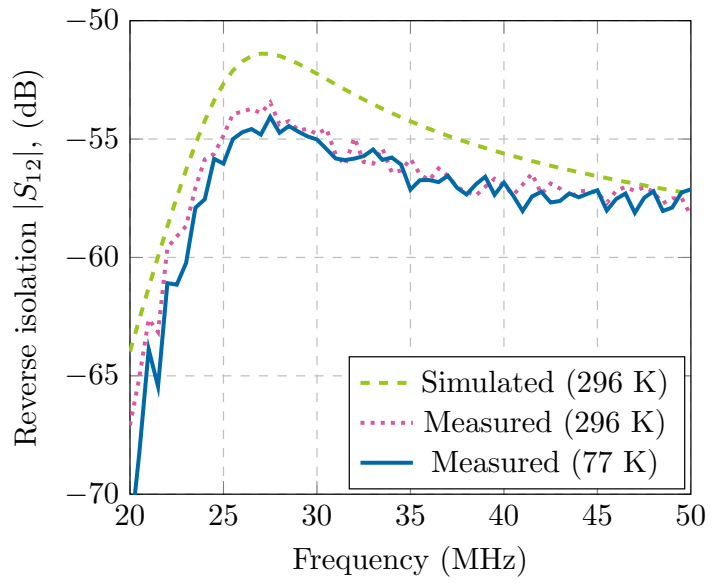

(b)

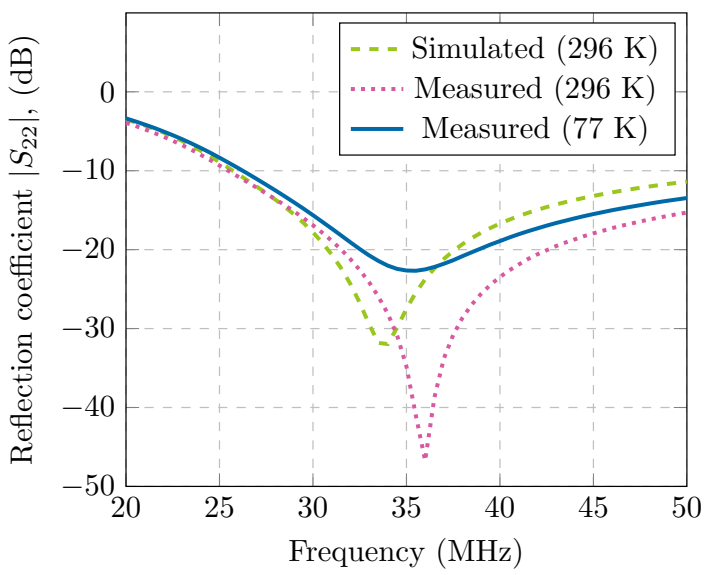

(d)

Figure 8. Measured and simulated S-parameters of the room temperature and cryogenic preamplifier.

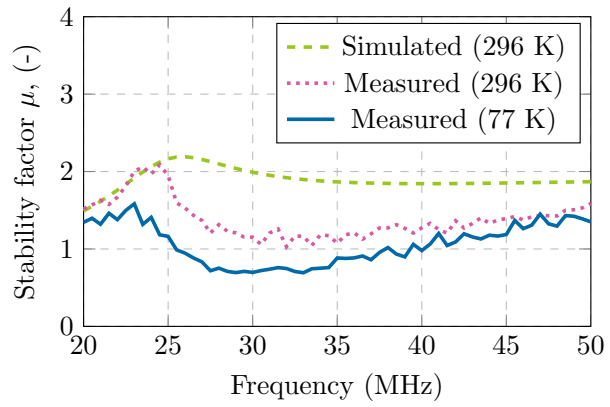

Figure 9. Simulated and measured stability factor of the preamplifier.

measured S-parameters and is plotted in Fig. 9. At room temperature the preamplifier is unconditionally stable both in simulation and measurement. However, when cryogenically cooled the $\mu$-factor drops below one. Hence the preamplifier is conditionally stable/potentially unstable at $77 \mathrm{~K}$. Since the preamplifier is potentially unstable, a second approach for determining stability is to measure the spectrum at the input (output) of the preamplifier while the output (input) is terminated by a short, open or load (the coil or $50 \Omega$ ).

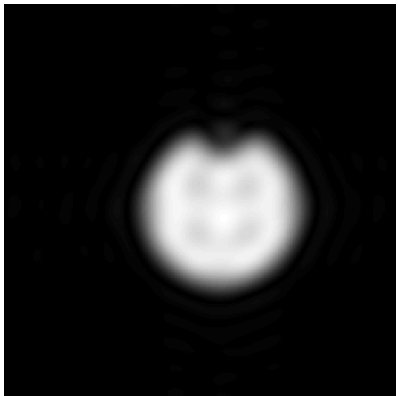

Figure 10. Measured magnitude image of the phantom using the cryogenic preamplifier cooled to $77 \mathrm{~K}$. The phantom is $37.5 \mathrm{~mm}$ in diameter. The fieldof-view is $120 \mathrm{~mm} \times 120 \mathrm{~mm}$. The image is interpolated from 16-by-16 to 256-by-256 pixels using zero-filling.

The measured spectrum shows no spurious signals indicating that the preamplifier is stable. In addition the current draw of the preamplifier is observed while the input and output is terminated by a short, open or load. When the current remains constant for all terminations, it is indirect indication that the preamplifier is stable. The last two approaches is basically a simplified source/load pull measurement focussed on stability. 
Table I

Measured Performance at $32 \mathrm{MHz}$

\begin{tabular}{ccccc}
\hline \hline Temperature & Noise [K] & Current [mA] & Gain [dB] & SNR \\
\hline $296 \mathrm{~K}$ & 35.4 & 49 & 23 & 365 \\
$77 \mathrm{~K}$ & 13.7 & 11 & 18 & 399 \\
\hline \hline
\end{tabular}

Hence, with the given combination of coil and matching circuit the preamplifier is stable. For this work, the preamplifier works sufficiently even with the potential instability. If unconditional stability is required a $200 \Omega$ shunt resistor can be added to the output of the preamplifier. This causes the $\mu$-factor to rise above unity while the gain drops by $1 \mathrm{~dB}$ to $17 \mathrm{~dB}$. Friis' formula for noise in cascaded two ports can be evaluated to determine if the gain drop is acceptable or not. For the application of MRI the gain drop is acceptable.

The measured noise is shown in Table I at $32 \mathrm{MHz}$. Cooling the preamplifier with liquid nitrogen decreases the noise by $20.7 \mathrm{~K}$, from $35.4 \mathrm{~K}$ to $13.7 \mathrm{~K}$. The current draw of preamplifier is also lowered by $38 \mathrm{~mA}$. The power consumption at room temperature is $147 \mathrm{~mW}$ and $33 \mathrm{~mW}$ at $77 \mathrm{~K}$. The gain also decreases by $5 \mathrm{~dB}$. This is expected due to freeze out and could be countered by increasing the biasing voltage. The noise simulated at room temperature yield $5.1 \mathrm{~K}$ relatively far from the $35.4 \mathrm{~K}$. This discrepancy between simulated and measured noise performance is primarily because the transistor model is based on measurements only above $2 \mathrm{GHz}$ and thus extrapolated downwards. Hence, the noise of the transistor model is not accurate when used at this, relatively, low frequency. Further, since the model is inaccurate, the noise matching might also be sub-optimal.

The required linearity is a $1 \mathrm{~dB}$ compression point better than $-20 \mathrm{dBm}$ [50], [51]. The $1 \mathrm{~dB}$ compression point of the preamplifier is measured at $-13.8 \mathrm{dBm}$ at both $296 \mathrm{~K}$ and $77 \mathrm{~K}$. Hence the preamplifier complies with the linearity requirement.

The image obtained in the scanner is seen in Fig. 10 using the cryogenic preamplifier. The image bears resemblance to an apple. This is due to the spherical phantom containing a small pocket of air. Due to the surface tension of the water this pocket of air seems to be impaled by another small pillar of liquid thus looking like the stem of an apple. In other words, the image looks as expected.

\section{DISCUSSION}

Although the frequency of operation was chosen at $32 \mathrm{MHz}$ due to scanner availability all methods discussed apply generally to MRI. With little work the presented preamplifier can be redesigned to another frequency by altering the input and output matching circuits.

An aspect in choosing components is their magnetic properties. Capacitors are available from e.g. Johnson or PPI and non-magnetic inductors are available from e.g. Coilcraft. Acquiring non-magnetic resistors is a more tedious process resulting in either long lead times or high cost. Hence magnetic resistors are used in the biasing of the preamplifier. The transistor is also not available in a non-magnetic package.
The magnetic packaging does disturb the main magnetic field, however the size of the components are small and they are placed approximately $30 \mathrm{~cm}$ away from the sample. No adverse effects were observed during imaging.

Concerning the conditional stability of the preamplifier at $77 \mathrm{~K}$ it is not inherently obvious looking at the S-parameter data. Since the input reflection coefficient approaches one the preamplifier must become more unstable as stated by (3). Lowering the gain, however, must increase stability. The reverse isolation stays constant and thus neither increase nor decrease stability. Hence, the decreased gain cannot offset the increase in input reflection coefficient. The conditionally stable preamplifier with a $\mu$-factor of 0.75 implies that when $\left|S_{22}\right|=\mu$ the preamplifier will oscillate. The impedance present at the output of the preamplifier is known to be $50 \Omega$ (or very close) because it is the impedance of the scanner RF ports. No problems with instability were observed.

The ATF54143 transistor was difficult to stabilize because it is designed primarily for $1-10 \mathrm{GHz}$ operation. Thus ensuring low frequency stability was difficult because the inherent gain in the transistor rises rapidly at lower frequencies. Hence internal feedback in the transistor cause oscillation unless the gain is lowered. The stability of the preamplifier is highly dependent on the layout. Especially parasitic inductance between source and ground can cause high frequency instability. Other low frequency transistors are not as prone to oscillation at high frequency. However, none were found that showed decent noise performance. Further, it is vital that the output and input are physically separated to not create a feedback path which cause instability.

The noise performance of the preamplifier is enhanced when cooled as expected. The relative SNR increase is approximately $8 \%$ when using a room temperature coil. However, as coil designs move towards cryogenic operation the noise of the preamplifier becomes increasingly dominant. Thus, if the coil is also cooled the SNR increase from using a cryogenic preamplifier becomes higher.

It is likely that even better noise performance can be achieved by more appropriate matching. This requires the characterization of the four noise parameters in order to find the optimum noise match. Indeed it is simpler to simulate the optimal noise match. However, the transistor model is extrapolated from $2 \mathrm{GHz}$ and downward and was not capable of simulation at $77 \mathrm{~K}$. Especially the transistor model needs revision for lower frequency and lower temperature. The passive components are also not characterized at cryogenic temperatures. Thus allowing simulations at cryogenic temperatures are contingent on proper characterization of the components used for design. Hence better models for cryogenic design is in demand.

\section{CONCLUSION}

The preamplifier design was based on the ATF54143 enhancement mode pHEMT using a common source topology. Bipolar transistors should generally not be considered for cryogenic work. Indeed HBTs can be made to work at cryogenic temperatures but requires custom design. On the 
other hand field effect transistors are much more likely to work at cryogenic temperatures. The results show that at liquid nitrogen the noise is $13.7 \mathrm{~K}$ whereas the gain drops to $18 \mathrm{~dB}$ from $23 \mathrm{~dB}$ at room temperature. This is expected due to the freeze out of carriers and can be corrected by increasing the bias voltage at the transistor gate. The SNR is increased from 365 to 399 using the cryogenic preamplifier with a room temperature coil.

An auxiliary system consisting of voltage regulator, trigger and switch circuits were presented. The supply regulator was designed using a readily available LM317 and ensures a stable, filtered supply voltage for the preamplifier. The trigger and switch ensures that the preamplifier does not break during transmission by switcing off the supply voltage to the preamplifier.

Moving towards the ultimate limit of detection cryogenic probes are seeing the light of day. Hence the noise contribution from the preamplifiers becomes more important. This work described the pitfalls of preamplifier design and implementation for cryogenic operation, including necessary auxiliary systems.

\section{REFERENCES}

[1] S. E. Day, M. I. Kettunen, F. A. Gallagher, D.-E. Hu, M. Lerche, J. Wolber, K. Golman, J. H. Ardenkjaer-Larsen, and K. M. Brindle, "Detecting tumor response to treatment using hyperpolarized 13C magnetic resonance imaging and spectroscopy," Nat. Med., vol. 13, no. 11, pp. 1382-1387, nov 2007.

[2] S. J. Nelson, J. Kurhanewicz, D. B. Vigneron, P. E. Z. Larson, A. L. Harzstark, M. Ferrone, M. van Criekinge, J. W. Chang, R. Bok, I. Park, G. Reed, L. Carvajal, E. J. Small, P. Munster, V. K. Weinberg, J. H. Ardenkjaer-Larsen, A. P. Chen, R. E. Hurd, L.-I. Odegardstuen, F. J. Robb, J. Tropp, and J. A. Murray, "Metabolic imaging of patients with prostate cancer using hyperpolarized [1-13C]pyruvate." Sci. Transl. Med., vol. 5, no. 198, pp. 1-22, aug 2013.

[3] D. Erdogmus, E. G. Larsson, R. Yan, J. C. Principe, and R. Fitzsimmons, "Measuring the signal-to-noise ratio in magnetic resonance imaging: a caveat," Signal Processing, vol. 84, pp. 1035-1040, 2004.

[4] R. E. Hurd, Y.-F. Yen, A. Chen, and J. H. Ardenkjaer-Larsen, "Hyperpolarized $13 \mathrm{C}$ metabolic imaging using dissolution dynamic nuclear polarization." J. Magn. Reson. Imaging, vol. 36, no. 6, pp. 1314-1328, dec 2012.

[5] E. Y. Chekmenev, "MRI Hyperpolarization and Molecular Imaging," Newsl. SNMMI, vol. 7, no. 3, pp. 2-4, 2013.

[6] C.-N. Chen and D. I. Hoult, Biomedical Magnetic Resonance Technology, 1st ed. Institute of Physics Publishing, 1989.

[7] D. G. Nishimura, Principles of Magnetic Resonance Imaging, 1st ed. lulu.com, 2010.

[8] H. Kovacs, D. Moskau, and M. Spraul, "Cryogenically cooled probes - a leap in NMR technology," Prog. Nucl. Magn. Reson. Spectrosc., vol. 46, pp. 131-155, 2005.

[9] L. Darrasse and J. Ginefri, "Perspectives with cryogenic RF probes in biomedical MRI," Biochimie, vol. 85, pp. 915-937, 2003.

[10] Bruker MRI CryoProbe. 2017. [Online]. Available: $\quad$ https://www.bruker.com/products/mr/preclinical-mri/mricryoprobes.html

[11] W. Kwok, Z. You, and J. Zhong, "Cryogenically cooled phased array rf receiver coil for magnetic resonance imaging," 2003, US Patent App. $10 / 405,633$.

[12] C.-1. Lim, P. Serano, and J. L. Ackerman, "Pre-amplifiers for a 15-Tesla magnetic resonance imager," in IEEE Int. RF Microw. Conf., 2013, pp. 295-299.

[13] S. H. Yadav, "Design of Low Noise Amplifier For MRI Scanner using Cascode Technology," Int. J. Innov. Res. Comput. Commun. Eng., vol. 5 , pp. 1504-1510, 2017.

[14] F. D. Doty, G. Entzminger, J. Kulkarni, K. Pamarthy, and J. P. Staab, "Radio frequency coil technology for small-animal MRI," NMR Biomed., vol. 20, no. 3, pp. 304-325, 2007.

[15] WanTCom Inc. 2017. [Online]. Available: http://www.wantcominc.com/

[16] Teledyne e2v (UK) Ltd. 2017. [Online]. Available: http://www.e2v.com/
[17] Hi-Q.A. Inc., Datasheet H1021531. 2011. [Online]. Available: http://www.hiqa.com/H1021531-Data Sheet.pdf

[18] M. W. Pospieszalski, "Extremely low-noise amplification with cryogenic FETs and HFETs: 1970-2004," IEEE Microw. Mag., vol. 6, no. 3, pp. $62-75,2005$.

[19] J. J. Bautista, "Cryogenic, Low-Noise High Electron Mobility Transistor Amplifiers for the Deep Space Network," The Telecommunications and Data Acquisition Report, Jet Propulsion Lab., California Inst. of Tech., Tech. Rep., pp. 65-80, 1993.

[20] U. Kleine, J. Bieger, and H. Seifert, "A Low-Noise CMOS Preamplifier Operating at 4.2 K," IEEE J. Solid-State Circuits, vol. 29, no. 8, pp. 921-926, 1994.

[21] R. J. Prance, A. P. Long, T. D. Clark, and F. Goodall, "UHF ultra low noise cryogenic FET preamplifier," J. Phys. E., vol. 15, pp. 101-104, 1982.

[22] A. T. Lee, "A low-power-dissipation broadband cryogenic preamplifier utilizing GaAs MESFETs in parallel," Rev. Sci. Instrum., vol. 64, pp. 2373-2378, 1993.

[23] S. Montazeri, W.-t. Wong, A. H. Coskun, and J. C. Bardin, "Ultra-LowPower Cryogenic SiGe Low-Noise Amplifiers: Theory and Demonstration," IEEE Trans. Microw. Theory Tech., vol. 64, no. 1, pp. 178-187, 2016.

[24] J. Koivuniemi and M. Krusius, "Noise temperature of cryogenic FET amplier with high-Q resonator," Phys. B, vol. 284-288, pp. 2149-2150, 2000.

[25] P. Styles and N. F. Soffe, "A High-Resolution NMR Probe in Which the Coil and Preamplifier Are Cooled with Liquid Helium," J. Magn. Reson., vol. 60, pp. 397-404, 1984.

[26] M. G. Richards, A. R. Andrews, C. P. Lusher, and J. Schratter, "Cryogenic GaAs FET amplifiers and their use in NMR detection," Rev. Sci. Instrum., vol. 57, pp. 404-409, 1986.

[27] G. Fontana, R. Mezzena, S. Vitale, and M. Cerdonio, "Improved Sensitivity of Planar Microwave Biased RF-SQUIDs using a Cryogenic HEMT Preamplifier," IEEE Trans. Appl. Supercond., vol. 3, no. 1, pp. $1820-1823,1993$

[28] R. Mathur, R. W. Knepper, and P. B. O'Connor, “A Low-Noise Broadband Cryogenic Preamplifier Operated in a High-Field Superconducting Magnet," IEEE Trans. Appl. Supercond., vol. 18, no. 4, pp. 1781-1789, 2008.

[29] J. G. Heteren, N. Fenzi, T. W. James, and L. C. Bourne, "Thin Film High Temperature Superconductor RF Coil and Cryogenic Preamplifier for Low Field MRI," in IEEE Nucl. Sci. Symp. Med. Imaging Conf., 1993, pp. 1708-1712.

[30] F. Resmer, H. C. Seton, and J. M. S. Hutchison, "Cryogenic receive coil and low noise preamplifier for MRI at 0.01 T," J. Magn. Reson., vol. 203, pp. 57-65, 2010.

[31] J. D. Sanchez-heredia, E. Søvsø, S. Hansen, C. Laustsen, V. Zhurbenko, and J. H. Ardenkjær-larsen, "Low-Noise Active Decoupling Circuit and its Application to 13C Cryogenic RF Coils at 3 T," Tomography, vol. 3 , no. 1 , pp. 60-66, 2017.

[32] LM317 3-Terminal Adjustable Regulator (SLVS044X), Texas Instruments, 2016.

[33] C. Hu, Modern Semiconductor Devices for Integrated Circuits. Prentice Hall, 2010. [Online]. Available: https://people.eecs.berkeley.edu/ hu/

[34] R. Pierret, Semiconductor Device Fundamentals. Addison-Wesley, 1996.

[35] - Advanced Semiconductor Fundamentals, 2nd ed. Pearson, 2003.

[36] B. Lengeler, "Semiconductor devices suitable for use in cryogenic environments," Cryogenics, vol. 14, pp. 439-447, 1974.

[37] J. D. Cressler, S. Member, J. H. Comfort, A. Member, E. F. Crabbc, G. L. Patton, S. Member, J. M. C. Stork, S. Member, J. Y. Sun, S. Member, and B. S. Meyerson, "On the Profile Design and Optimization of Epitaxial Si- and SiGe-Base Bipolar Technology for $77 \mathrm{~K}$ Applications - Part I: Transistor DC Design Considerations," IEEE Trans. Electron Devices, vol. 40 , no. 3, pp. $525-541,1993$.

[38] J. D. Cressler, S. Member, E. F. Crabbc, J. H. Comfort, A. Member, J. M. C. Stork, S. Member, J. Y. Sun, and S. Member, "On the Profile Design and Optimization of Epitaxial Si- and SiGe-Base Bipolar Technology for 77 K Applications - Part II: Circuit Performance Issues," IEEE Trans. Electron Devices, vol. 40, no. 3, pp. 542-556, 1993.

[39] J. Cressler, "Operation of SiGe bipolar technology at cryogenic temperatures," J. Phys., vol. 4, pp. C6-101-C6-110, 1994.

[40] E. F. Crabbe, G. L. Patton, J. M. C. Stork, J. H. Comfort, B. S. Meyerson, and J. Y. C. Sun, "Low temperature operation of Si and SiGe bipolar transistors," in International Technical Digest on Electron Devices, Dec 1990, pp. 17-20. 
[41] E. F. Crabbe, D. L. Harame, B. S. Meyerson, J. M. C. Stork, and J. Y. C. Sun, "Low-temperature operation of sige p-n-p hbts," IEEE Trans. Electron Devices, vol. 39, no. 11, pp. 2638-2639, Nov 1992.

[42] S. Weinreb, J. C. Bardin, and H. Mani, "Design of Cryogenic SiGe LowNoise Amplifiers," IEEE Trans. MTT, vol. 55, no. 11, pp. 2306-2312, 2007.

[43] M. Pospieszalski, S. Weinreb, and R. Norrod, "FET's and HEMT's at Cryogenic Temperatures- Their Properties and Use in Low-Noise," IEEE Trans. MTT, vol. 36, no. 3, pp. 552-560, Mar 1988.

[44] H. Meschede, J. Albers, R. Reuter, J. Kraus, D. Peters, W. Brockerhoff, and F. J. Tegude, "Rf investigations on hemt's at cryogenic temperatures down to $20 \mathrm{k}$ using an on-wafer microwave measurement setup," in European Microwave Conference, vol. 1, Sept 1992, pp. 151-156.

[45] D. Johansen, J. Sánchez-Heredia, V. Zhurbenko, and J. ArdenkjærLarsen, "Practical Aspects of Preamplifier Designs for 13C Imaging," in Proc. Intl. Soc. Mag. Reson. Med. 25, Abstract 4299, 2017.

[46] M. L. Edwards and J. H. Sinsky, "A new criterion for linear 2-port stability using a single geometrically derived parameter," IEEE Trans. MTT, vol. 40, no. 12, pp. 2303-2311, Dec 1992.

[47] J. Rollett, "Stability and Power-Gain Invariants of Linear Twoports," IRE Transactions on Circuit Theory, vol. 9, no. 1, pp. 29-32, March 1962.

[48] F. Teyssandier and D. Prêle, "Commercially Available Capacitors at Cryogenic Temperatures," in WOLTE9, Jun. 2010.

[49] R. D. Norrod, "Cryogenic Measurements of Surface Mount Multi-layer Ceramic Chip Capacitors," National Radio Astronomy Observatory, Electronics Division, Green Bank, West Virginia, Tech. Rep. 214, March 2010.

[50] R. Behin, J. Bishop, and R. M. Henkelman, "Dynamic range requirements for MRI," in Proc. Intl. Soc. Mag. Reson. Med. 13, pp. 845, 2005.

[51] — - "Dynamic range requirements for MRI," Concepts in Magnetic Resonance Part B: Magnetic Resonance Engineering, vol. 26B, no. 1, pp. 28-35, 2005.

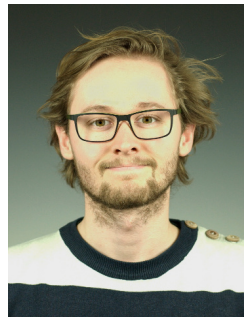

Daniel H. Johansen received his M.Sc. in Wireless Communication Systems, cum laude, from Aalborg University in 2013. In 2016 he joined the Center for Hyperpolarization in Magnetic Resonance and Electromagnetic Systems at the Technical University of Denmark as a $\mathrm{PhD}$ student. Before joining DTU, he designed reconfigurable antennas at RFMD and electronics for the IoT at Sigma Designs. His work focuses on the design and implementation of cryogenic preamplifiers, single and array coils for magnetic resonance imaging.

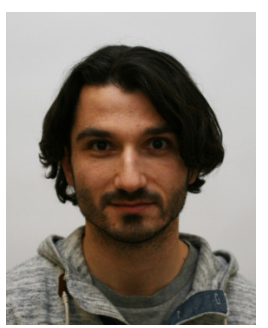

Juan D. Sanchez-Heredia received his M.Sc. degree from the University of Murcia in 2010, and the $\mathrm{Ph}$.D. degree from the Technical University of Cartagena, both in Telecommunications Engineering. In 2014 he joined the Center for Hyperpolarization in Magnetic Resonance at the Technical University of Denmark, where he is currently a postdoc. His research interests include the development of multiantenna systems for wireless communications, application of coil arrays to low gamma nuclei MRI, low noise MRI electronics and high-field MRI coils.

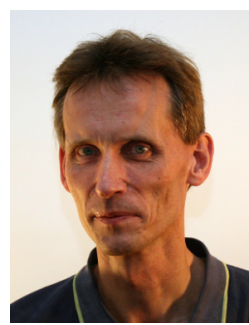

Jan R. Petersen received his M.Sc. and Ph.D. degrees in electrical engineering from the Technical University of Denmark, Denmark, in 1990 and 1994, respectively. In 2015 he joined the Electromagnetic Systems group, DTU Elektro, Technical University of Denmark, Denmark, where he is currently a Development Engineer. Current research and teaching interests covers analog LF/RF electronics, in the form of electronics for sensors and measurements systems, including instrument and system control with LabVIEW.

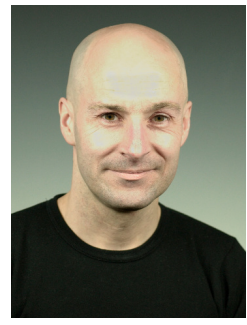

Tom K. Johansen received his M.Sc. and Ph.D. degrees in electrical engineering from the Technical University of Denmark, Denmark, in 1999 and 2003 , respectively. In 1999 he joined the Electromagnetic Systems group, DTU Elektro, Technical University of Denmark, Denmark, where he is currently an Associate Professor. From September 2001 to Marts 2002 he was a Visiting scholar at the center for wireless communication, University of San Diego, California. He has spent external research stays at the Ferdinand Braun Institute (FBH), in Berlin, Germany. His research areas include the modeling of high-frequency solid-state devices, millimeter-wave and sub-millimeter-wave integrated circuit design, and reconfigurable microwave filter design.

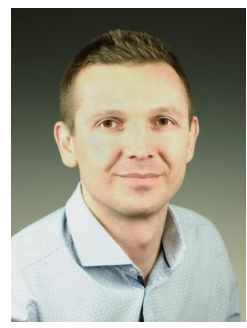

Vitaliy Zhurbenko received his M.Sc. degree from the Kharkiv National University of Radio Electronics in 2001, and the Ph.D. degree from the Technical University of Denmark in 2008, all in Electrical Engineering. In 2005, he joined the Technical University of Denmark, where he is currently an Associate Professor. His present research interests include microwave and millimeter wave sensing for biomedical and security applications; microwave and millimeter-wave components and integrated circuits for instrumentation applications; antennas and passive circuit design and characterization; magnetic resonance technology.

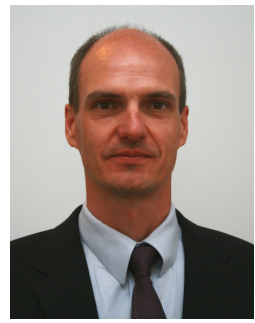

Jan H. Ardenkjær-Larsen received his M.Sc. and $\mathrm{Ph} . \mathrm{D}$. degrees in electrical engineering from the Technical University of Denmark in 1991 and 1995 respectively. He is currently a full professor and head of Center for Magnetic Resonance at the Technical University of Denmark. He is also center leader for Center for Hyperpolarization in Magnetic Resonance, a center of excellence funded by the Danish National Research Foundation. His research has always evolved around means to improve the sensitivity of magnetic resonance imaging and spectroscopy, with the aim of obtaining new, and otherwise inaccessible, information about function and disease at the cellular and molecular levels. 